\title{
Path Planning and Workspace Determination for Robot-Assisted Insertion of Steerable Electrode Arrays for Cochlear Implant Surgery
}

\author{
Jian Zhang ${ }^{1}$, Wei Wei ${ }^{1}$, Spiros Manolidis ${ }^{2}$, J. Thomas Roland $\mathrm{Jr}^{3}$, \\ and Nabil Simaan ${ }^{1, *}$ \\ ${ }^{1}$ Department of Mechanical Engineering, Columbia University \\ Laboratory of Advanced Robotics and Mechanism Applications \\ RM 234 S.W. Mudd Building, 500 West $120^{\text {th }}$ Street, New York, NY 10027, USA \\ ${ }^{2}$ Department of Head \& Neck Surgery, Beth Israel Hospital \\ ${ }^{3}$ Department of Otolaryngology and Neurosurgery, New York University Medical Center \\ \{jz2181,ww2161, ns2236@columbia.edu, smanolidis@mac.com, \\ tom.roland@med.nyu.edu \\ http: / /www.columbia.edu/cu/mece/arma /
}

\begin{abstract}
In previous works, the authors showed that using robot-assisted steerable electrode array insertions can significantly reduce the insertion forces compared to non-steerable electrode arrays. In addition to steering the electrode array, it is possible to change its angle of approach with respect to the scala tympani. This paper focuses on determining the relevance of changing the angle of approach of the electrode array by comparing steerable electrode array insertions using a two Degreesof-Freedom (DoF) robot versus a four DoF robot. Optimal insertion path planning strategies are presented for both two and four DoF insertions. Simulation results and experiments show that the four DoF insertions can improve over two DoF insertions. Moreover, changing the angle of approach can further reduce the insertion forces. The simulation results also provide the workspace requirements for designing a custom parallel robot for robot-assisted cochlear implant surgery.
\end{abstract}

\section{Introduction}

Patients suffering from severe hearing loss, but with intact auditory nerves, can regain partial hearing through cochlear implant surgery. The cochlear implant system, Fig. 1, includes a microphone, a micro-processor, a transmitter, a receiver, and an electrode array. The microphone and processor convert sound waves into electrical signals that are wirelessly transmitted to a receiver embedded in the mastoid bone. These electrical signals are then used to excite

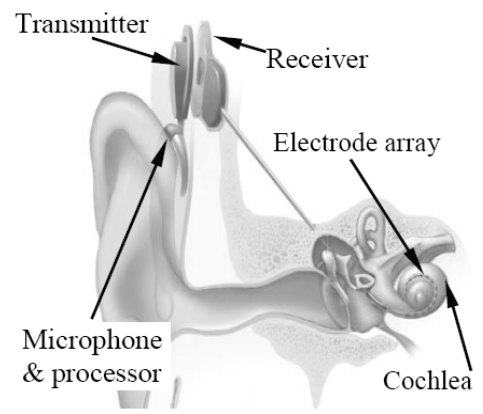

Fig. 1. Cochlear implant system

\footnotetext{
${ }^{*}$ Corresponding author.
} 
specific electrodes in the electrode array. These electrodes correspond to different sound frequencies that can be restored by direct excitation of the auditory nerves.

The anatomic structure of the cochlea and its cross section are shown in Fig. 2. In cochlear implant surgery, the long, thin, and flimsy electrode array is carefully inserted into the scala tympani or scala vestibuli. Earlier works, e.g. [1], focused on insertions of the electrode array into the scala vestibuli. This approach was abandoned in favor of scala tympani insertions.

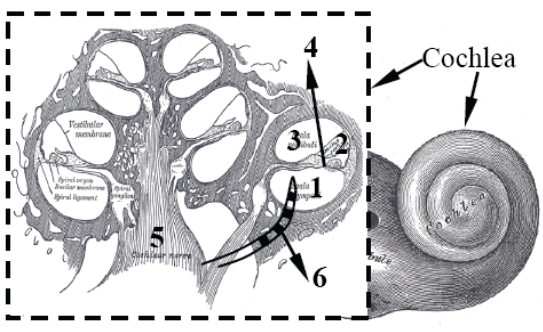

Fig. 2. Cochlea anatomic structure
1. Scala tympani
2. Scala media
3. Scala vestibule
4. Basilar membrane
5. Auditory nerve
6. Electrode array Regardless of the approach used, it is evident from previous works [2-6] that the current electrode insertions cause intracochlear trauma. This is due to lack of real-time imaging assistance, lack of force feedback during the insertion process, and the lack of controllability of the flexible electrode arrays.

In order to reduce trauma, various electrode arrays have been designed. Flexible and coiled electrode array designs have been proposed by MedEl Corp. (FLEX ${ }^{\text {soft }}$ Electrode), Cochlear Inc. (Contour Advance Electrode), and Advanced Bionics (HiFocus Helix Electrode). These electrode arrays are designed to passively bend to accommodate the curvature of the scala tympani during insertions.

In [7], a pilot study on robot-assisted insertion of novel steerable electrode arrays was conducted. These steerable electrode arrays were actuated using an actuation wire embedded in a silicone rubber electrode array. This work showed that using steerable electrode arrays and robotic insertions can significantly reduce the insertion forces. Subsequently, [8] used nitinol shape memory alloy wires embedded inside the electrode array to provide steerability.

Previous work [7] used steerable electrode arrays and a two DoF robot that is capable of controlling the insertion depth and the bending (steering) of the electrode array. In addition to bending the electrode arrays, it is possible to change its angle of approach with respect to the scala tympani.

Fig. 3 shows a representation of our experimental setup. It can be used as a four DoF robot or as a two DoF robot. In the case where four DoF are used, the angle of approach, the insertion depth, and the bending of the electrode array are controlled during insertion. In the case where only two DoF are used, only the insertion depth and the bending of the electrode array are controlled.

This paper extends the work in [7] by quantifying the importance of changing the angle of approach of steerable electrode arrays. Optimal path planning algorithms are presented to provide the optimal bending and the angle of approach of the steerable electrode array. These algorithms

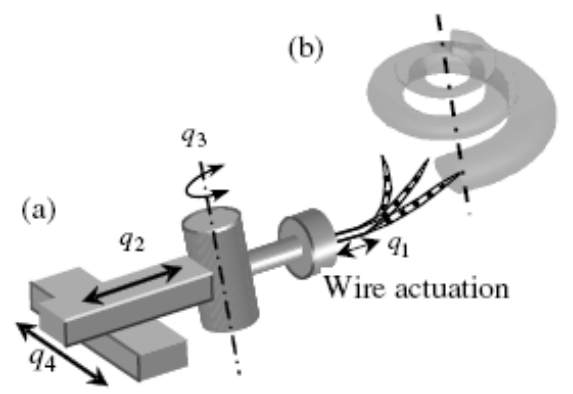

Fig. 3. (a) Four DoF insertion system (b) scala tympani model 
are simulated for determining the required workspace of a custom-designed robot for steerable electrode array insertions. Further, experiments are conducted to validate the efficacy of our approach.

Section 2 provides the mathematical modeling for the calibration and the optimal insertion path planning of the steerable electrode array. Section 3 simulates and compares two DoF with four DoF electrode insertions. Section 4 shows the experimental results comparing two and four DoF electrode insertions.

\section{Modeling and Path Planning of Two and Four DoF Insertions}

Image-based electrode calibration: An optimal insertion path planning strategy reduces the intracochlear trauma and facilitates insertions. We assume that reducing the insertion forces is correlated with decreased risk of electrode array buckling and electrode tip migration outside of the scala tympani. The approach in [7] called for minimizing the shape discrepancy between the steerable electrode array and the scala tympani in order to minimize the required insertion forces. In this section, we extend this approach to four DoF electrode insertions.

The calibration process of the steerable electrode array was described in [7] based on manually digitized images of selected points along the backbone of the steerable electrode array. This tedious process is replaced in Fig. 4 by an automatic edge detection and curve fitting algorithm that yields a closed-form approximation to the shape of the electrode array. For any given bent shape of the steerable electrode array, a picture is first converted to black and white and then a Canny filter is applied to detect the edges of the electrode array. Each edge is approximated by a fourth order polynomial curve and the backbone of the electrode array is calculated by averaging both edges.

A series of $z$ pictures, Fig. 5, are taken in order to calibrate a given steerable electrode array. Picture $j$ is associated with a given amount of pull $q_{1 j}$ on the actuation wire. For each picture, a polynomial model of the backbone of the steerable electrode array is fitted according to Eq. (1), where $\theta\left(s, q_{1 j}\right)$ represents the angle of the electrode array tangent at arc length $s$ for $q_{1 j}$.

$$
\begin{aligned}
& \text { Vectors } \quad \boldsymbol{\psi}(s)=\left[1, s, s^{2}, s^{3}, s^{4}\right]^{T} \\
& \mathbf{a}_{j}\left(q_{1 j}\right)=\left[1, q_{1 j}, q_{1 j}{ }^{2}, q_{1 j}{ }^{3}, q_{1 j}{ }^{4}\right]^{T}
\end{aligned}
$$
repre-

and Fig. 4. Electrode array edge detection: (a) Original picture (b) Black and white (c)
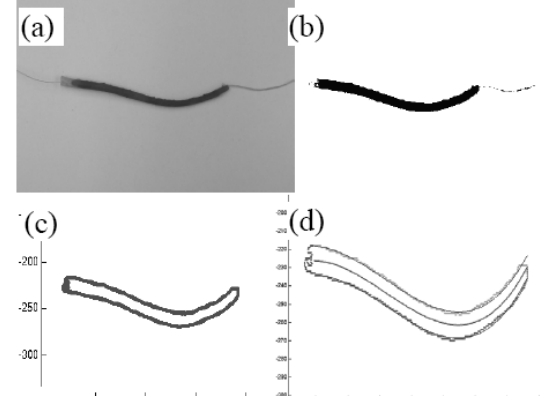

Canny filter (d) Backbone curve fitting

sent the fourth-order polynomial approxi-

mation to the bending angle of the electrode array. To solve for the two parameter optimization problem, a least squares solution is applied in order to find the polynomial coefficients vector $\mathbf{a}_{j}\left(q_{1_{j}}\right)$. This result is a point $\mathbf{a}_{j}\left(q_{1_{j}}\right) \in \mathfrak{R}^{5}$ for each picture $j(j=1,2, \ldots, z)$. 


$$
\theta_{j}\left(s, q_{1 j}\right)=\sum_{p=0}^{4} a_{j, p} s^{p}=\boldsymbol{\psi}^{T}(s) \mathbf{a}_{j}\left(q_{1 j}\right) \quad j=1,2, \ldots, z
$$

Next, a second interpolation over vectors $\mathbf{a}_{j}\left(q_{1 j}\right)$ 's is carried out using cubic splines. Following [9], we define $k$ intervals (segments) $\left\lfloor\mathbf{a}_{k}\left(q_{1 k}\right), \mathbf{a}_{k+1}\left(q_{1 k+1}\right)\right\rfloor$ where $k=1,2, \ldots, z-1$. For any given $q_{1} \in\left[q_{1_{k}}, q_{1_{k+1}}\right\rfloor$, Eq. (2) solves for the coefficients of the cubic spline for segment $k$; then the shape of the electrode is given by Eq. (3), where $\mathbf{c}_{k}\left(q_{1}\right)$ and $\mathbf{B}_{k}$ are given by Eqs. (4) and (5). In Eq. (4), $\mathbf{a}_{k}^{\prime}$ denotes the derivative of $\mathbf{a}_{k}\left(q_{1}\right)$ with respect to $q_{1}$.

$$
\begin{gathered}
\mathbf{a}_{k}\left(q_{1}\right)=\mathbf{B}_{k}{ }^{T} \mathbf{c}_{k}\left(q_{1}\right) \\
\theta_{k}\left(s, q_{1}\right)=\psi^{T}(s) \mathbf{B}_{k}{ }^{T} \mathbf{c}_{k}\left(q_{1}\right) \\
q_{1 j=k} \leq q_{1} \leq q_{1=k+1}, \quad 1 \leq k \leq z-1 \\
\mathbf{c}_{k}\left(q_{1}\right)=\left[1, q_{1}, q_{1}{ }^{2}, q_{1}{ }^{3}\right]^{T}
\end{gathered}
$$$$
\mathbf{B}_{k}=\left[\begin{array}{cccc}
1 & 0 & 0 & 0 \\
0 & 1 & 0 & 0 \\
-3 / q_{1_{k+1}}^{2} & -2 / q_{1_{k+1}} & 3 / q_{1_{k+1}}^{2} & -1 / q_{1_{k+1}} \\
2 / q_{1_{k+1}}^{3} & 1 / q_{1_{k+1}}^{2} & -2 / q_{1_{k+1}}^{3} & 1 / q_{1_{k+1}}^{2}
\end{array}\right]\left[\begin{array}{c}
\mathbf{a}_{k} \\
\mathbf{a}_{k}^{\prime} \\
\mathbf{a}_{k+1} \\
\mathbf{a}_{k+1}^{\prime}
\end{array}\right]
$$

Fig. 5. Calibration images of the steerable electrode array (a) deep insertions (b) shallow insertions

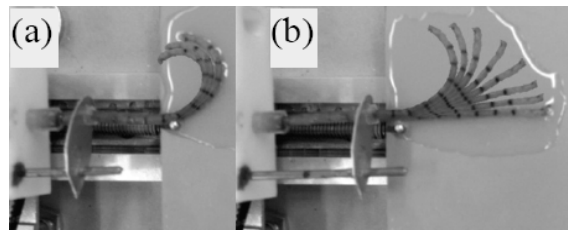

Insertion path planning: In [7], we presented the optimal path planning for two DoF electrode insertions. For brevity, in this paper we only include the path planning for four DoF electrode insertions, Fig. 3. In Fig. 3, the approach angle of the electrode array with respect to the scala tympani is denoted by $q_{3}$ and the electrode actuation wire pull is denoted by $q_{1}$. The translation components of the gripper holding the electrode array are given by $q_{2}$ and $q_{4}$. A

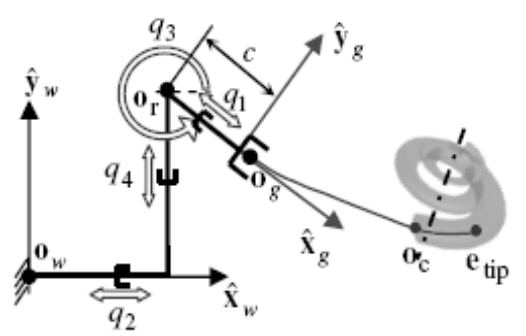

Fig. 6. Kinematic model of a four DoF robot

kinematic representation of the robot and the electrode array is given in Fig. 6.

For any given insertion depth $d$, the optimal values of $q_{1}$ and $q_{3}$ are obtained by minimizing the objective function in Eq. (6), which is an extension of the approach in [7]. This function minimizes the shape discrepancy between the steerable electrode array and the scala tympani model using interpolation of the experimental data results to find the global minimum. $L$ represents the overall length of the electrode array, and $\theta_{c}\left(s_{c}\right)$ stands for the angle of the curve tangent of the scala tympani at arc length $s_{c}$. 


$$
\underset{q_{1}, q_{3}}{\arg \min } \int_{L-d}^{L}\left(\theta\left(\tau, q_{1}\right)-\theta_{c}(\tau-(L-d))+q_{3}(d)\right)^{2} d \tau
$$

Once $q_{1}$ and $q_{3}$ are found, the required translation DoF $q_{2}$ and $q_{4}$ are easily found by Eq. (7) using the inverse kinematics of the robot in Fig. 6.

$$
\left[\begin{array}{l}
q_{2} \\
q_{4}
\end{array}\right]=\mathbf{o}_{g}\left(q_{1}, q_{3}\right)-\left[\begin{array}{l}
c \cos q_{3} \\
c \sin q_{3}
\end{array}\right]
$$

where $c$ is the length from $\mathbf{o}_{g}$ (position of the robot gripper) to $\mathbf{o}_{r}$ (rotation center).

\section{Simulation and Workspace Determination}

Fig. 7. shows the simulation results of optimal path planning for four DoF insertions using the steerable electrode array of Fig. 5. Fig. 8 compares the two DoF with four DoF insertions based on these simulation results and it shows that the four DoF insertions fit the shape of the scala tympani better than the two DoF insertions. This result is confirmed by experiments in section 4 .
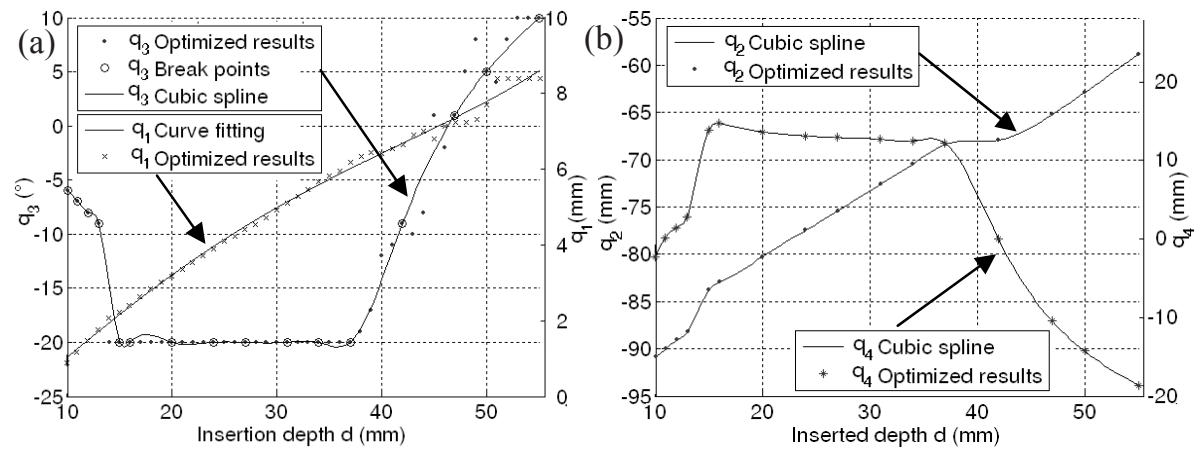

Fig. 7. Path planning for: (a) $q_{1}$ and $q_{3}$ (b) $q_{2}$ and $q_{4}$
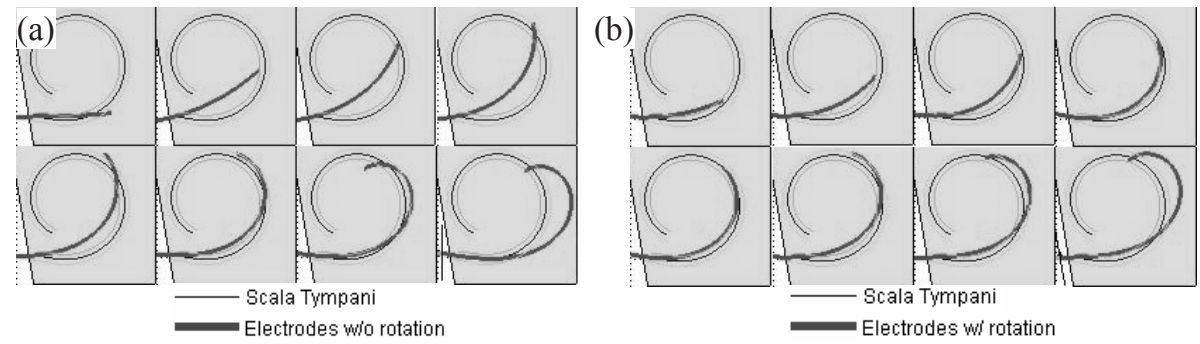

Fig. 8. Insertion simulation: (a) two DoF insertions (b) four DoF insertions 
The simulation results of Fig. 7 were carried out for the 3:1 scaled-up steerable electrode array of Fig. 5. From Fig. 7, the required robot joint values vary within the intervals $q_{3} \in\left[-20^{\circ}, 10^{\circ}\right], q_{2} \in[-91,-58] \mathrm{mm}$, and $q_{4} \in[-18,15] \mathrm{mm}$. The required joint ranges are thus $33 \mathrm{~mm}, 30^{\circ}$, and $33 \mathrm{~mm}$ for $q_{2}, q_{3}, q_{4}$ respectively. If scaled down properly, these results provide the required workspace for robot-assisted insertion of true size (1:1) steerable cochlear implant electrode arrays.

In Fig. 6, the distance between the revolute joint of the robot $\mathbf{o}_{r}$ and the electrode tip $\mathbf{e}_{t i p}$ for the 3:1 electrode array is $110 \mathrm{~mm}$. In real application, the estimated depth of full insertions is about $27 \mathrm{~mm}$ measured from the cochleostomy point $\mathbf{o}_{c}$ to the electrode tip $\mathbf{e}_{t i p}$. The average distance from the cochleostomy point $\mathbf{o}_{c}$ to the opening in the skull is $60 \mathrm{~mm}$ for adults. We increase this number by $10 \mathrm{~mm}$ for safety. Hence the required distance from $\mathbf{o}_{r}$ to $\mathbf{e}_{t i p}$ of a true size (1:1) steerable electrode array is estimated to be $97 \mathrm{~mm}$.

Since the robot rotates the electrode while keeping the cochleostomy point $\mathbf{o}_{c}$ fixed during insertion, it is easy to scale down the required workspace of the robot. Theoretically the scaling can be done while considering the electrode array as a rigid body rotating about $\mathbf{o}_{c}$. Since the distance between $\mathbf{o}_{r}$ and $\mathbf{o}_{c}$ varies during insertion, we choose to use the full length of the electrode to provide a conservative estimate for the required workspace (i.e. we use the condition $\mathbf{o}_{c}=\mathbf{e}_{\text {tip }}$ ). To scale down the required workspace for a 1:1 steerable electrode, a scaling factor of $97 / 110$ is directly applied to the translational joints of the robot. The resulting required joint ranges are $30 \mathrm{~mm}$ for $q_{2}$ and $q_{4}$. The minimal required rotation range for joint $q_{3}$ is not scaled down and it remains $30^{\circ}$.

We are currently using this workspace estimation to design a miniature Stewart-Gough

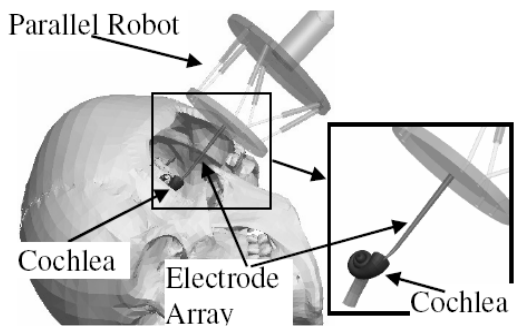

Fig. 9. Potential parallel robot insertion setup CAD model parallel robot for electrode array insertion. Our stated design goals for this robot are $\pm 20^{\circ}$ tilting about any axis and $\pm 20 \mathrm{~mm}$ translation in all directions. The parallel robot type was chosen for its compactness, precision, and portability [10].

\section{Experimental Validation}

Experimental setup: Fig. 8 shows that changing the angle of approach of the electrode array with respect to the scala tympani allows decreasing the shape discrepancies between the steerable electrode array and the scala tympani. These simulation results were based on the calibration and the simulation path planning for the steerable electrode in Fig. 5. The same steerable electrode was used for two DoF 
and four DoF insertion experiments using the experimental setup of Fig. 10. The goal of this experiment is to validate the correlation between the results in Fig. 8 and the reduction of the electrode insertion forces when four DoF insertion path planning is used compared to only two DoF insertions.

During the experiments, the scala tympani channel was wetted by glycerin to emulate the environment inside the cochlea. The robot of Fig. 10 was controlled with Linux Real Time Application Interface (RTAI) with a closed loop rate of $1 \mathrm{kHz}$. An AG NTEP 5000d single axis force sensor was used in the setup to measure the axial insertion force of the steerable electrode array during the whole insertion process and it was capable of detecting $\pm 0.1 \mathrm{~g}$ force using a 13 bits $\mathrm{A} / \mathrm{D}$ acquisition card. Each insertion was repeated three times to validate the repeatability of the results. Fig. 11 shows how the approach angle of the electrode array is changed with respect to a planar scala tympani model during four DoF insertions. The force reading data of these experiments are shown in Fig. 12.

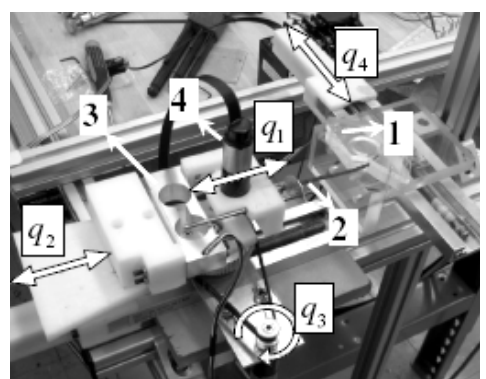

Fig. 10. Experimental setup: 1. Planar scala tympani model 2. Steerable electrode array 3. Single axis force sensor 4. Motor for the steerable electrode array

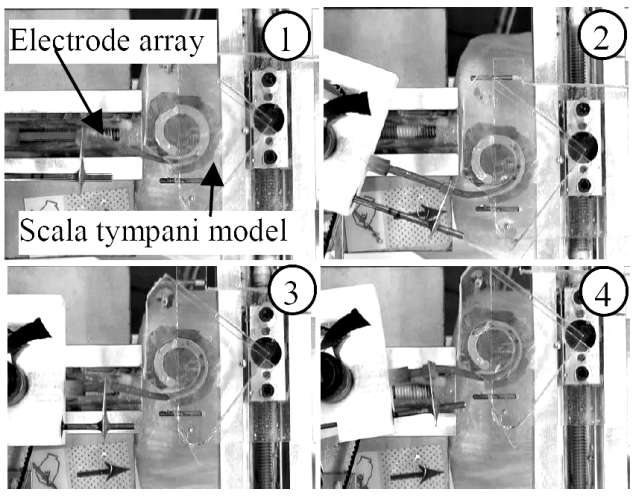

Fig. 11. Experiment of a four DoF steerable electrode insertion

Results: Fig. 12 (a) shows the results of two DoF steerable electrode array insertions. The average insertion force for the three experiments is 0.7 grams. In comparison, the four DoF insertions in Fig. 12 (b) had an average insertion force of 0.40 grams, $43 \%$ smaller than two DoF robot insertions. The peak of force happens when the electrode array first hits the outer wall of the scala tympani model and therefore generates the maximal insertion force. For two DoF insertions, the maximal peak value among three experiments is 4.8 grams. While four DoF insertions keep the peak value as 3.9 grams, $19 \%$ less than the two DoF insertions peak. The negative forces happen in Fig. 12 (a) and (b) because the steerable electrode hugs the inner wall of the scala tympani [11]. These results suggest the effectiveness of adjusting the angle of approach of the steerable electrode array with respect to the scala tympani in reducing the insertion forces. 

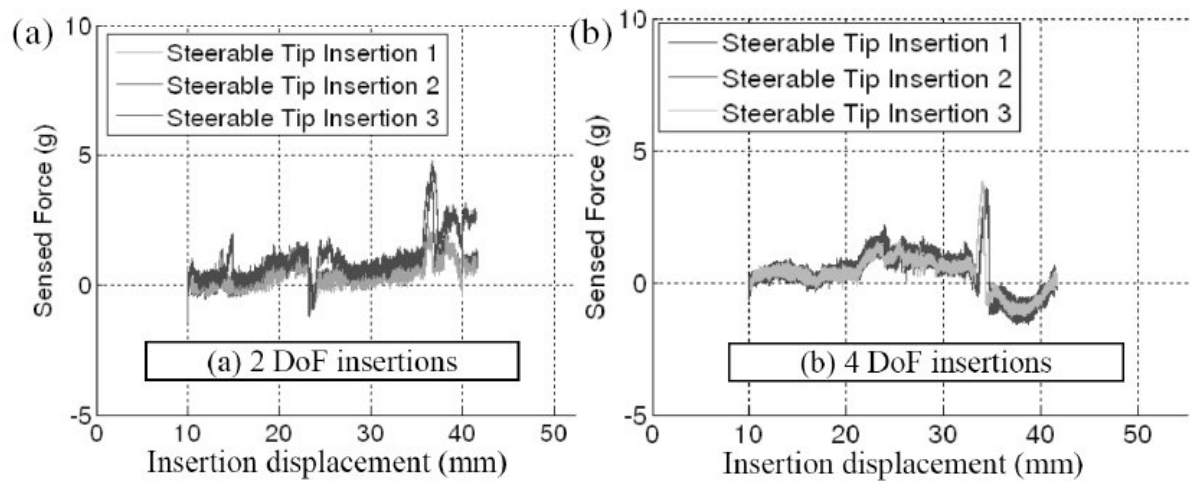

Fig. 12. Experimental results: (a) two DoF insertions (b) four DoF insertions

\section{Conclusion}

Our previous work [7] proved the efficacy of using steerable electrode arrays in reducing insertion forces compared to non-steerable electrode arrays. This work extends these results by testing the hypothesis that changing the angle of approach of the steerable electrode array with respect to the scala tympani can further reduce the insertion forces. An image-based algorithm for steerable electrode array calibration and path planning for four DoF electrode array insertions (insertions that control steerability and the angle of approach) were presented. Simulations showed that the four DoF insertions decrease the shape discrepancy between the steerable electrode array and the scala tympani better than two DoF insertions. It also provided necessary workspace for robot-assisted cochlear implant insertions. Experiments validated our simulation results and showed that changing the angle of approach may reduce the average insertion forces by up to $40 \%$ and the peak force by $19 \%$. Using these results we are designing a custom parallel robot for steerable electrode array insertions in cochlear implant surgery.

Acknowledgement. This research is supported by Grant \#0651649 from National Science Foundation (CBET).

\section{References}

1. Kiefer, J., et al.: Scala vestibuli Insertion in Cochlear Implantation: A Valuable Alternative for Cases with Obstructed Scala tympani. ORL 62(5), 251-256 (2000)

2. Maeno, T., Hiromitsu, S., Kawai, T.: Control of grasping force by detecting stick/slip distribution at the curved surface of an elastic finger, San Francisco, CA, USA. Institute of Electrical and Electronics Engineers Inc, Piscataway (2000)

3. Wardrop, P., et al.: A temporal bone study of insertion trauma and intracochlear position of cochlear implant electrodes. II: Comparison of Spiral Clarion and HiFocus II electrodes. Hear. Res. 203(1-2), 68-79 (2005) 
4. Gstoettner, W., et al.: Cochlear implant deep electrode insertion: extent of insertional trauma. Acta. Otolaryngol. 117(2), 274-277 (1997)

5. Roland, J.T.J.: A model for cochlear implant electrode insertion and force evaluation: results with a new electrode design and insertion technique. Laryngoscope 15(8), 1325-1339 (2005)

6. Fishman, A., Roland Jr., J.T., Alexiades, G., Mierzwinski, J., Cohen, N. L.: Fluoroscopically assisted cochlear implantation. Otol. Neurotol. 24(6), 882-886 (2003)

7. Zhang, J., et al.: A Pilot Study of Robot-Assisted Cochlear Implant Surgery Using Steerable Electrode Arrays, in Medical Image Computing and Computer-Assisted Intervention MICCAI 2006. In: Larsen, R., Nielsen, M., Sporring, J. (eds.) MICCAI 2006. LNCS, vol. 4190, pp. 33-40. Springer, Heidelberg (2006)

8. Chen, B., Kha, H., Clark, G.: Development of a steerable cochlear implant electrode array. In: 3rd Kuala Lumpur International Conference on Biomedical Engineering, pp. 607-610 (2007)

9. Rogers, D.F., Adams, A.J.: Mathematical Elements for Computer Graphics, 2nd edn. McGraw-Hill, New York (1990)

10. Simaan, N., Shoham, M.: Robot Construction for Surgical Applications. In: 1st IFAC Conference on Mechatronics Systems, Darmstadt, Germany (2000)

11. Todd, C.A., Naghdy, F., Svehla, M.J.: Force Application During Cochlear Implant Insertion: An Analysis for Improvement of Surgeon Technique. IEEE Transactions on Biomedical Engineering 54(7), 1247-1255 (2007) 